Recepción: 20 / 04 / 2017

Aceptación: 20 / 05 / 2017

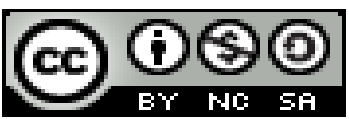

Ciencias Sociales y políticas

Publicación: 15 / 07 / 2017

\title{
Obtención y cálculo de los impuestos municipales, a través del estado financiero anual
}

\section{Collection and calculation of municipal taxes, through the annual financial statement}

\section{Cobrança e cálculo de impostos municipais, através da demonstração financeira anual}

\author{
Jhonny A. Concha- Ramírez ${ }^{\mathrm{I}}$ \\ jhonnyconcharamirez@gmail.com \\ Fátima M. Fernández-Flores ${ }^{\mathrm{II}}$ \\ fatimar_1881@hotmail.com \\ Carmen K. Barros- Merizalde ${ }^{\mathrm{III}}$ \\ kathybarros@live.com \\ Correspondencia: fatimar_1881@hotmail.com
}

\footnotetext{
I. Diploma Superior en Tributación; Ingeniero en Sistemas Administrativos Computarizados; Ingeniero en Contaduría Pública y Auditoria CPA; Instituto Tecnológico Superior Babahoyo, Ecuador.

II. Magister en Gerencia de Servicios de Salud; Magister en Administración de Empresas con mención en Recursos Humanos; Diploma Superior en Gestión del Talento Humano; Diploma Superior en Gestión de Desarrollo de los Servicios de Salud; Ingeniera Comercial; Instituto Tecnológico Superior Babahoyo, Ecuador.

III. Magister en Administración de Empresas; Ingeniera Comercial; Instituto Tecnológico Superior Babahoyo, Ecuador.
} 


\section{Resumen}

La recaudación tributaria se sustenta en permanentes controles que debe realizar el estado por medio de la administración tributaria. Es así que posee diversas maneras de recaudar tributo. No todos los contribuyentes y en especial las personas naturales están al tanto de las normas que regulan la tributación, ocasionando de esta manera que exista un grave desconocimiento de los derechos y obligaciones de los contribuyentes que se perjudican por el pago de intereses y multas. El desarrollo de este diseño trata de cómo ASERNAVAL S.A. debe hacer para obtener y pagar sus tributos seccionales a través de sus Estados Financieros, en la forma y manera adecuada y sin demora, para evitar sanciones de parte de la administración municipal, pagar a terceros que realicen sus trámites municipales y poder obtener todos los permisos sin necesidad de perder tiempo y dinero.

Palabras claves: Impuestos; estados financieros; patente; tasa de habilitación; municipio. 


\section{Abstract}

The tax collection is based on permanent controls that must be carried out by the state through the tax administration. So it has different ways of collecting tribute. Not all taxpayers and especially natural persons are aware of the rules that regulate taxation, causing in this way a serious ignorance of the rights and obligations of taxpayers who are prejudiced by the payment of interest and fines. The development of this design is about how ASERNAVAL S.A. Must do to obtain and pay its sectional taxes through its Financial Statements, in the proper and prompt manner, to avoid penalties on the part of the municipal administration, to pay third parties that perform their municipal procedures and to obtain all the permits Without the need to waste time and money.

Key words: Taxes; financial statements; patent; habilitation rate; municipality. 


\section{Resumo}

A arrecadação de impostos é baseada em controles permanentes a serem executadas pelo Estado através da administração fiscal. Assim, ele tem várias maneiras de aumentar impostos. Nem todos os contribuintes e pessoas especialmente naturais estão cientes das regras que regem a tributação, causando, assim, há um desconhecimento grave dos direitos e obrigações dos contribuintes que são prejudicadas pelo pagamento de juros e multas. O desenvolvimento deste projeto é como ASERNAVAL S.A Você deve fazer para obter e pagar os seus impostos seccionais através de suas Demonstrações Financeiras, da maneira e de forma adequada e sem demora, para evitar sanções por parte do governo local, pagar terceiros para conduzir os seus procedimentos municipais e obter todas as autorizações sem perder tempo e dinheiro.

Palavras-chave: Impostos; demonstrações financeiras; patentes; permitindo taxa; municipio. 


\section{Introducción.}

ASERNAVAL S.A. fue constituida en la ciudad de Guayaquil, el 21 de julio de 2000, inscrita en el registro mercantil el 14 de Agosto de 2000 Inició su actividad el 28 de Agosto de 2000. La compañía, sólo cuenta con un establecimiento situado en la ciudad de Guayaquil y tiene como actividad principal la venta de equipo de seguridad.

Debido a un cese de actividades en 2007 la compañía reapertura sus actividades en el año 2010, estando pendiente de pago de todos los impuestos, es entonces donde se ve la necesidad de pagar los impuestos municipales, requisito indispensable para entrar a un concurso de proveedores de equipos de seguridad.

El principal problema de la compañía ASERNAVAL S.A., es que no cuenta con la persona adecuada para el control y organización de los trámites e impuestos que tiene que realizar dicha institución, por cuanto el personal con el que cuenta la compañía es bastante reducido.

Las personas con las que cuenta la empresa no están capacitadas para realizar los trámites, no saben que tienen que hacer, ni las fechas en las que se tienen que presentar la documentación, ni cuáles son los pasos a seguir para realizar los trámites municipales, es por eso que la compañía se encuentra atrasada en estos impuestos.

Todo nace a raíz de que se constituyó la compañía, nunca se preocuparon por los impuestos municipales dándole mayor énfasis solo en los impuestos tributarios del SRI, eso hizo que a la fecha de hoy no se tenga ningún permiso municipal, y esto hace que se tenga que contar con un gran capital de dinero para cubrir los gastos municipales, cosa que no se tiene.

El principal problema de la compañía ASERNAVAL S.A. es que no posee una guía de cómo realizar los trámites para obtener los permisos municipales, ni de cómo calcularlos, ni que requisitos 
necesita para ello, y en qué fecha se deben de tramitar para estar al día y no caer en mora, evitando recibir notificaciones de clausura del local donde se realiza sus actividades diarias de trabajo.

Obtener y calcular los impuestos municipales, utilizando el balance general, para conocer el verdadero valor a pagar en las ventanillas de recaudación del municipio de Guayaquil.

Distinguir cuales son las principales fuentes de ingresos de los municipios, las fuentes de la obligación tributarias municipales y la clasificación de los impuestos municipales.

El proyecto es de gran importancia porque además de servir de guía a la compañía ASERNAVAL S.A., sirve de guía y es aplicable a todas las sociedades privadas y no solo eso sino que también sirven para las personal naturales obligadas a llevar contabilidad pues así pagan sus impuestos municipales, tales como 1.5 por mil, tasas como patente y tasa de habilitación y demás trámites.

En el Ecuador, los tributos son partes del presupuesto general del Estado. Los ingresos tributarios a la fecha de hoy han aumentado por ello se han convertido en un pilar fundamental para el presupuesto nacional, lo que ha hecho que el Estado ha buscado la manera de obligar a los contribuyentes a liquidar oportunamente los respectivos impuestos.

Para la realización de este proyecto se planteó un presupuesto, cumplido a cabalidad por la empresa y el equipo de investigación.

\section{Materiales y métodos.}

La técnica que se usó en el desarrollo de este proyecto principalmente es la observación, ya que el problema a investigarse corresponde a la sociedad y fue seleccionado únicamente después de algunas sugerencias sobre las complicaciones que tienen la persona obligada a llevar contabilidad al realizar sus declaraciones de impuestos municipales. 
Como segunda técnica utilizada está la entrevista, ya que para la información recopilada necesitaba algunas opiniones de profesionales que hayan pasado o estén pasando en la actualidad por problemas parecidos al ejemplo expuesto y para esto entrevisté a algunos de ellos y obtuve información necesaria que me ayudó con el desarrollo del proyecto, la misma que se llevó a cabo con la utilización de instrumentos de la investigación, el más importante es el cuestionario.

Todo el desarrollo de esta investigación nos lleva únicamente a la obtención de resultados, que serán aplicados para solucionar un problema que se ha vuelto tan común en la sociedad como es la declaración de impuesto municipales.

\section{Resultados.}

Se ha realizado una encuesta a los trabajadores de ASERNAVAL S.A. con el fin de conocer la situación general de conocimiento tributario y su importancia dentro de la empresa. Los resultados interpretados de estas entrevistas arrojan el siguiente panorama:

Las personas que trabajan en esta organización en su mayor parte son personas profesionales capaces de entender la problemática por cual atraviesa la empresa. Las personas encargadas de la administración solo ocupan el 20\%, mientras el resto del personal el $80 \%$, por eso se tiene que tener presente que la administración es la encargada de los tributos municipales y no hay una persona capacitada para realizar dicha tarea.

Del total de la muestra el $80 \%$ respondió saber poco de lo que son los tributos municipales y un $20 \%$ respondió no saber. Este porcentaje tan alto nos muestra un gran desconocimiento sobre la declaración de los tributos municipales. El 20\% sabe que es el Impuesto a la Renta, el 50\% esta inseguro y el $30 \%$ no sabe de qué se trata. 
Al $10 \%$ parece importarle los tributos municipales, el $30 \%$ no opina mucho y el $60 \%$ no pone importancia. La mayoría (90\%) no se preocupa por las fechas de declaraciones de los pagos de los impuestos y la persona que lo sabe no avisa con anticipación la fecha para las declaraciones, desconoce que existen sanciones por no declarar impuestos, no sabe cuáles pasos seguir para la declaración y pago de los tributos municipales, no sabe de dónde se deben tomar los datos para declarar los tributos municipales, consideran poco necesario o innecesario provisionar los valores a pagar por impuestos, desconoce que el municipio le otorga a la empresa una tasa de habilitación de funcionamiento de local para el desarrollo de sus actividades. El 70\% no tiene conocimientos sobre cuáles son los requisitos para realizar el pago de los tributos municipales. 
Obtención y cálculo de los impuestos municipales, a través del estado financiero anual

\section{Cuadro 1. Plan de Ejecución}

\begin{tabular}{|c|c|c|c|c|c|}
\hline \# & OBJETIVOS ESPECÍFICOS & ACTIVIDADES & RECURSOS & \multicolumn{2}{|c|}{ PRESUPUESTO } \\
\hline 1 & $\begin{array}{l}\text { Identificar los hechos generadores de los } \\
\text { tributos municipales para lograr determinar su } \\
\text { procedencia y una correcta aplicación de las } \\
\text { normas constitucionales, legales y } \\
\text { reglamentarias. }\end{array}$ & $\begin{array}{l}\text { Capacitar al personal sobre la importancia de los tributos } \\
\text { Revisar las ordenanzas municipales } \\
\text { Conocer las leyes que facultan a los municipios a aplicar los } \\
\text { tributos. }\end{array}$ & $\begin{array}{l}\text { Instructor } \\
\text { Libros } \\
\text { resaltador }\end{array}$ & $\begin{array}{l}\$ \\
\$ \\
\$\end{array}$ & $\begin{array}{r}200,00 \\
60,00 \\
3,00\end{array}$ \\
\hline 2 & $\begin{array}{l}\text { Analizar y comprender que es el } 1.5 * \text { mil, } \\
\text { patente, su aplicación como herramienta } \\
\text { tributaria y la importancia de las mismas. }\end{array}$ & $\begin{array}{l}\text { Aplicar los artículos de la ley, ordenanzas para su correcto } \\
\text { calculos } \\
\text { Comprobar que cumplimos con los requisitos necesarios } \\
\text { Adquirir la tasa municipal para empezar el tramite }\end{array}$ & $\begin{array}{l}\text { Archivador } \\
\text { Hojas } \\
\text { Tasa Municipal }\end{array}$ & $\begin{array}{l}\$ \\
\$ \\
\$\end{array}$ & $\begin{array}{l}2,00 \\
1,00 \\
2,00\end{array}$ \\
\hline 3 & $\begin{array}{l}\text { Comprender la importancia de la Tasa de } \\
\text { Habilitación para el funcionamiento del giro } \\
\text { del negocio. }\end{array}$ & $\begin{array}{l}\text { Realizar el cálculo de los impuestos necesarios } \\
\text { Cumplir con todos los requisitos necesarios } \\
\text { Llenar formulario especial para este tramite }\end{array}$ & $\begin{array}{l}\text { Calculadoras } \\
\text { Tasa Municipal } \\
\text { Boligrafo }\end{array}$ & $\begin{array}{l}\$ \\
\$ \\
\$\end{array}$ & $\begin{array}{r}10,00 \\
6,00 \\
0,50\end{array}$ \\
\hline 4 & $\begin{array}{l}\text { Conocer y analizar los requisitos básicos } \\
\text { para la obtención de los tributos municipales. }\end{array}$ & $\begin{array}{l}\text { Presentar El formulario } 101 \text { en la supertintendencia de } \\
\text { compañias } \\
\text { Tener la declaracion de impuesto a la renta Formulario } 101 \\
\text { del SRI } \\
\text { Llenar formularios de } 1,5 \text { * mil y patente y tasa de } \\
\text { habilitacion dado por el municipio }\end{array}$ & $\begin{array}{l}\text { Fotocopias } \\
\text { Impresiones } \\
\text { varios }\end{array}$ & $\$$ & 10,00 \\
\hline 5 & $\begin{array}{l}\text { Determinar y liquidar la carga tributaria a } \\
\text { pagar por los diversos tributos municipales en } \\
\text { aplicación de las respectivas ordenanzas } \\
\text { municipales }\end{array}$ & $\begin{array}{l}\text { Acercarnos a las ventanillas del Municipio. } \\
\text { Entregar los requisitos } \\
\text { Esperar en la ventanilla hasta que tabulen la informacion y } \\
\text { poder pagar los tributos }\end{array}$ & Movilización & $\$$ & 6,50 \\
\hline & & Son: Trescientos treinta y un $00 / 100$ dolares & TOTAL & $\$$ & 331,00 \\
\hline
\end{tabular}

\section{Cuadro 2. Cronograma}

\begin{tabular}{|c|c|c|c|c|c|c|c|c|c|c|c|c|c|}
\hline \multirow{3}{*}{$\mathbf{N}^{\circ}$} & \multirow{3}{*}{ TIEMPO } & \multicolumn{12}{|c|}{2011} \\
\hline & & \multicolumn{4}{|c|}{ ENERO } & \multicolumn{4}{|c|}{ FEBRERO } & \multirow[b]{2}{*}{$1 \mathrm{~S}$} & \multirow[b]{2}{*}{$2 S$} & \multirow[b]{2}{*}{$3 \mathrm{~S}$} & \multirow[b]{2}{*}{ 4S } \\
\hline & & $1 \mathrm{~S}$ & $2 S$ & $3 S$ & 4S & $1 \mathrm{~S}$ & $2 S$ & $3 \mathrm{~S}$ & $4 S$ & & & & \\
\hline 1 & $\begin{array}{l}\text { Conseguir la Ley, Reglamento y Código } \\
\text { Tributario. }\end{array}$ & $\mathbf{x}$ & & & & & & & & & & & \\
\hline 2 & Analizar los Artículos & $\mathbf{x}$ & & & & & & & & & & & \\
\hline 3 & Ir a municipios a ver requisitos & $\mathbf{X}$ & & & & & & & & & & & \\
\hline 4 & Comprar diferentes tasas para tramites & & $\mathbf{X}$ & & & & & & & & & & \\
\hline 5 & Llenar los formularios del municipio & & $\mathbf{X}$ & & & & & & & & & & \\
\hline 6 & Sacar permiso de bomberos & & $\mathbf{X}$ & & & & & & & & & & \\
\hline 7 & Obtener permiso uso de suelo & & & $\mathbf{X}$ & & & & & & & & & \\
\hline 8 & Obtener tasa de desechos sólidos & & & & $\mathbf{X}$ & & & & & & & & \\
\hline 9 & Llenar balance (For. 101 SRI) & & & & $\mathbf{x}$ & & & & & & & & \\
\hline 10 & Pagar Formulario 101 SRI & & & & & $\mathbf{X}$ & & & & & & & \\
\hline 11 & Presentar y sellar form. 101 SRI a la Super. Cias. & & & & & & $\mathbf{X}$ & & & & & & \\
\hline 12 & Llenar form. $1,5^{*}$ mil y patente & & & & & & $\mathbf{x}$ & & & & & & \\
\hline 13 & Llevar form. 101 y requisitos al municipio & & & & & & $\mathbf{x}$ & & & & & & \\
\hline 14 & $\begin{array}{l}\text { Presentar Form. SRI y Municipio para pagar } \\
\text { tributos }\end{array}$ & & & & & & $\mathbf{x}$ & & & & & & \\
\hline 15 & Pagar tributos en el Municipio de Guayaquil & & & & & & & $\mathbf{X}$ & & & & & \\
\hline 16 & Iniciar Tramite para Tasa de Habilitación & & & & & & & $\mathbf{X}$ & & & & & \\
\hline 17 & Obtener Tasa de Habilitación y Control de Local & & & & & & & & $\mathbf{X}$ & $\mathbf{x}$ & $\mathbf{X}$ & $\mathbf{X}$ & $\mathbf{X}$ \\
\hline
\end{tabular}

Fuente: Ing. Jhonny Concha Ramirez 


\section{Requisitos para acercarse a realizar el trámite del pago del tributo municipal}

- Impuesto a la renta (copia certificada por la superintendencia de compañías).

- $\quad$ Permiso de bomberos.

- $\quad$ Pago de tasa de tramite $1.5 *$ mil.

- $\quad$ Llenar el formulario de impuestos municipales dado por el municipio.

- $\quad$ Copia RUC.

- Copia nombramiento representante legal.

- Copia cedula y votación del representante legal.

\section{¿Cuál es el procedimiento para declarar los tributos municipales?}

Cumplir con los requisitos antes mencionados, ir a las ventanillas del municipio en el departamento financiero de la ventanilla 22 a la 29, y esperar a que le den los valores a pagar.

Sí se realiza mal el impuesto a la renta (formulario 101) se debería que corregir tal impuesto y volverlo a presentar en la superintendencia de compañías para su nueva certificada y volver hacer todo el trámite de cálculos y pagos de los tributos.

La consecuencia de no cumplir con los impuestos municipales es el cierre parcial del local de funcionamiento de la compañía por parte del municipio, multas y citaciones.

Del Balance General que es un estado financiero, que va inmerso en la declaración del impuesto a la renta formulario 101 del SRI, es de donde se obtienen la mayor parte de la información para la declaración de los tributos municipales.

Se recomienda siempre hacer la declaración en los primeros 3 meses del año entrante, pagar los tributos municipales, así también se evitara largas colas de espera. 
Estas fechas van de acuerdo con el tipo de tributo municipal a pagar, desde enero hasta mayo, plazo en el cual no se harán recargos por multa o sea por interés al tributo a pagar.

\section{Ordenanza municipal aplicable al cantón Guayaquil}

Base Legal: Ordenanza de defensa s/n. R.O. 494, VIERNES 31 DE DICIEMBRE DEL 2004

\section{Plazo para el pago del Impuesto de Patente}

Art.5.- Este impuesto se pagara hasta 30 días de la fecha límite establecida para la declaración del impuesto a la renta.

\section{Obligaciones de los Sujetos Pasivos}

Art. 6.- Los sujetos pasivos del impuesto de patente están obligados a cumplir con los deberes formales establecidas en el Código Tributario, en todo cuanto se relaciona con este impuesto, y especialmente con los siguientes:

1) Inscribirse en el catastro de impuesto de patente que para la determinación de este impuesto llevara la Dirección Financiera Municipal.

2) Presentar la declaración del capital con que operen, en los formularios entregados por la Administración Tributaria Municipalidad, proporcionada los datos necesarios relativos a su actividad; y, comunicar oportunamente los cambios que se operen.

3) Llevar los libros y registros contables relacionados con la actividad económica que ejerzan, de conformidad con las disposiciones establecidas en la ley de régimen tributario interno, como en su reglamento. 
4) Facilitar a los funcionarios autorizados por la administración tributaria municipal las inspecciones o exhibiendo las declaraciones, informes, libros, registros y documentos pertinentes para tales efectos y formular las declaraciones que les fueran solicitadas.

5) Concurrir a las oficinas de la Dirección Financiera Municipal, cuando su presencia sea requerida por esta.

\section{Base Imponible para determinar el Impuesto de Patente}

Art. 8.- La base del impuesto anual de patente será en función del capital con el que operan los sujetos pasivos de este impuesto dentro del cantón, del año inmediato anterior, de acuerdo a las declaraciones que se deben presentar a la Superintendencia de Compañías o Superintendencia de Bancos, en el caso de personas jurídicas

\section{Tarifa del Impuesto}

Art. 9.- Sobre la base imponible en la forma prevista en el artículo anterior se establece el impuesto anual de patente de la siguiente manera: 


\section{Cuadro 3. Impuesto Anual de Patente}

\begin{tabular}{|c|c|}
\hline VALOR CAPITAL DE OPERACION & $\begin{array}{c}\text { VALOR A PAGAR POR EL IMPUESTO DE } \\
\text { PATENTE }\end{array}$ \\
\hline $\begin{array}{c}\text { Los negocios que operan con el capital de hasta } \\
\$ 12.500\end{array}$ & $\begin{array}{c}\text { \$10.00 es la base del pago } \\
\text { Quienes operen con un capital mayor a } \$ 12.501\end{array}$ \\
$\begin{array}{c}\text { El monto máximo a pagar, para el año 2005, será } \\
\text { de } \$ 5000\end{array}$ \\
\hline
\end{tabular}

Fuente: Hansen-Holm, Manual de obligaciones tributarias 2009, p, 216.

\section{Documentación Requerida}

- $\quad$ Formulario "solicitud para Registro de patente personas Jurídicas “

- Original (para verificación) y copia de los estados unidos financieros del periodo inmediato anterior al año de registro dela patente municipal, con la fe de presentación de la superintendencia de compañías o bancos, según sea el caso.

- $\quad$ Original y copia legible de la cedula de ciudadanía y del nombramiento actualizado del representante legal.

- Copia del R.U.C. actualizado de la compañía.

- $\quad$ copia de la escritura de constitución (cuando es por 1ra vez)

- $\quad$ copia legible del comprobante de pago de patente del año anterior.

- $\quad$ original (para verificación) y copia legible de la declaración y comprobante de pago del impuesto del 1.5 por mil del año correspondiente (para renovación de patente ) 


\section{Documentación Adicional}

- $\quad$ Original y copia legible de tasa por servicio de prevención de incendios (otorgado por el benemérito de bomberos), para establecimientos de concurrencia pública masiva (bares, discotecas, restaurantes, hoteles, cines, teatros, salas de espectáculos públicos, etc.)

\section{Nota:}

Cuando se va realizar este trámite por primera vez, se debe presentar al formulario: "solicitud para registro de patente personas jurídicas"

Cuando se trate de renovación de patente, se deberá presentar el formulario: "declaración conjunta del impuesto del 1.5 por mil y registro de patente municipal para personas obligadas a llevar contabilidad

\section{Tramite de Declaraciones y pago}

1) Solicitar el formulario "registro de patente municipal personas jurídicas" en las ventanillos de patente de la dirección financiera (Bloque noroeste 2). La tasa de trámite para el pago de patentes (cuyo valor es de \$2.00)se incluirá en el comprobante de pago de dicho impuesto

2) llenar la solicitud con la letra imprenta o a máquina.

3) Adjuntar los requisitos indicados a la solicitud y entregar en las ventanillas de patente de la dirección financiera, bloque Noroeste 2, se le entregara la liquidación correspondiente. No se aceptara documentación incompleta o legible.

4) cancelar en las ventanillas de recaudaciones (bloque noroeste 1) el valor del impuesto

5) el trámite de renovación se lo deberá realizar anualmente. El plazo para presentar este formulario es hasta el 30 de junio. Entendiendo que las personas obligadas a llevar contabilidad sean 
estas naturales o jurídicas se desglosa el procedimiento a seguir para el pago de tributos municipales.

En cuanto a lo legal están respaldados por La ley de Régimen Tributario Interno, el Reglamento para la aplicación de la Ley de Régimen Tributario Interno, el Código Tributario y La ley Orgánica de Régimen Municipal.

En cuanto a la parte técnica el estudio se basa en las ordenanzas municipales, ya que aquí están dados todos los valores a pagar por los tributos.

\section{Conclusiones.}

- De acuerdo a los resultados de la encuesta podemos concluir que la mayoría de las personas que trabajan en esta compañía son profesionales que no tienen mucho conocimiento sobre los tributos municipales, por ende no le prestan la atención debida al asunto.

- $\quad$ No existe una persona encargada especialmente a los tributos, ni que tampoco es delegada a nadie de los trabajadores presentes, y las personas encargadas de la administración no tienen presente sus obligaciones municipales.

- Que los profesionales encuestados, solo ven su trabajo personal y no le prestan atención a los permisos en general que la compañía necesita para su funcionamiento.

\section{Recomendaciones}

Capacitar al personal de administración sobre los tributos municipales y hacerle ver la importancia que tienen para el funcionamiento de las actividades diarias de la compañía.

- Contratar un especialista externo solo para que realice este trámite si no existiera personal interno, esto sería solo en la fecha exacta de las declaraciones para el pago de los tributos, el cual resultaría más económico para la compañía. 
- Tener siempre presente las fechas a declaras de los tributos para evitar sanciones, económicas como de cierre.

- Declarar a tiempo el impuesto a la renta al SRI (formulario 101), ya que con este formulario se debe declarar los tributos municipales.

- Evitar omitir valores en el formulario de impuesto a la renta, ya que esto haría que los tributos municipales se tengan que volver a calcular y declarar, esto traería un retraso en los permisos municipales.

\section{Bibliografía.}

Benites Chiriboga, Mayte y LASSO, Jorge Fernando, “Diplomado En Tributación, Introducción A La Tributación", editorial de la UTPL, 1ra edición, 4ta reimpresión, Loja-Ecuador, 2009.

Bernal Torres, Cesar Augusto, "metodología de la Investigación para administración, economía, humanidades, ciencias sociales", Pearson Educación, 2da edición, México, 2006.

Cuesta Álvarez, Rafael, “Auditoria de Estados Financieros”, Edino 2000, Guayaquil-Ecuador 2010.

González, Eusebio y LEJEUNE, Ernesto, “derecho tributario 1", plaza universitaria ediciones, salamanca, 3ra edición, 2003.

Hansen-Holm, "Manual De Obligaciones Tributarias", Distribuidora de texto del Pacifico S.A., 3ra edición, Ecuador, 2009.

Izquierdo Arellano, Enrique, "Investigación Científica 5 métodos y diseños de investigación”, Imprenta Cosmos, 12da edición, Loja-Ecuador 2007.

Jarrín, Pedro Pablo, “Guía práctica de Investigación Científica”, I.S.B.N., 3era edición, Quito-Ecuador, 2001.

Leiva, Francisco, “Nociones de Metodología de Investigación Científica”, Quito-Ecuador, 1988.

Pacheco Gil, Oswaldo, “Investigación III”, Mundiciencia, Guayaquil-Ecuador 2007.

Queralt, Juan Martin y Otros, "curso de derecho financiero tributario", 14ta edición, Editorial Tecnos, España, 2003.

Salkind, Neil, “método de la investigación”, Pearson Educación, México, 3era edición, 1999.

Troya Jaramillo, José Vicente, “estudios de derecho tributario”, serie de estudios jurídicos, volumen 1, corporación editora nacional, Quito, 1984. 
Villalba Avilés, Carlos, "Guía para la elaboración de anteproyectos y proyectos”, Sur Editores, Ecuador, 5ta edición, 2009. 